\title{
Commentary Testing the Gateway Hypothesis
}

\author{
Michael L Miller' and Yasmin L Hurd*,I \\ 'Departments of Psychiatry and Neuroscience, Friedman Brain Institute, Icahn School of Medicine at Mount Sinai, New York, NY, USA
}

Neuropsychopharmacology (2017) 42, 985-986; doi:I0.1038/npp.2016.279; published online I8 January 2017

The gateway drug hypothesis refers to the pattern of substance use during adolescence whereby legal substances, such as nicotine and alcohol, precede the progressive use of illicit substances like cocaine and heroin. This concept of a gateway progression related to addiction vulnerability has had important implications with respect to biology, society, and public policy. The gateway drug hypothesis was initially formulated by epidemiological studies which, aiming to characterize the natural history of substance use, were not designed to address the issue of 'causality' and therefore garnered substantial controversy. Specifically, opponents argued that since the initial studies could not distinguish between a drug's direct effect and other confounding variables (eg, environment), the term gateway drug was misleading. To address such criticisms, controlled animal experiments over the past few decades have helped to provide causal neurobiological insights relevant to the gateway hypothesis and drug addiction vulnerability.

Fredriksson et al (2016), being a recent example, tested the gateway hypothesis by assessing the effect of alcohol exposure on future cocaine use disorder in self-administering rats. Based on a comprehensive set of experiments, the researchers ultimately found that exposure to moderate or high amounts of alcohol beginning in late adolescence/early adulthood (and extending for 7 weeks) does not impact subsequent cocaine self-administration when examined later in adulthood. This study importantly builds on previous animal studies that, as opposed to assessing self-administration, had measured behavioral proxies of addiction such as novelty-seeking and conditioned place preference. The current findings nicely contribute to the field's growing knowledge base, but the multifaceted complexity of substance use disorder requires several factors to be kept in mind that, if incorporated into future experiments, may address remaining questions that thoroughly evaluate the gateway drug hypothesis.

\footnotetext{
*Correspondence: Dr YL Hurd, Departments of Psychiatry and Neuroscience, Friedman Brain Institute, Icahn School of Medicine at Mount Sinai, One Gustave L. Levy Place, Box 1639, New York, NY 10029, USA, Tel: +I 212824 8314, Fax: +I 6465279598 , E-mail: yasmin.hurd@mssm.edu

Received 27 November 2016; revised 2 December 2016; accepted 8 December 2016
}

Given that the brain's vulnerability to a drug is dynamic, and affected by age, one of the most important gateway considerations is the developmental period of drug exposure. Fredriksson et al (2016) started alcohol administration on either postnatal day 44 or 64, and these ages-roughly corresponding to late adolescence and early adulthood, respectively, in humans-were selected because they matched the onset of most binge drinking behavior. Although binge drinking does occur late, the predisposing pattern of substance use described by the gateway drug hypothesis refers to the initial and continued casual use of a substance, which occur during early to mid-adolescence and therefore precedes binge drinking behavior. The importance of age of onset to potential gateway sensitivity is illustrated by studies showing that drug exposure during adulthood does not alter the subsequent sensitivity to a particular substance as it does if administered during the adolescent period (Spear, 2016).

Another interesting factor is the interaction between the specific exposure type and the drug-class whose vulnerability is altered. Although evidence supporting a gateway relationship between alcohol and psychostimulants remains mixed and controversial, the literature supports a relatively consistent association between early alcohol exposure (either adolescent or gestational) and future alcohol consumption (Spear, 2016). The notion of drug-drug specificity applies to other drug classes as well, with studies consistently suggesting that adolescent nicotine exposure sensitizes an individual to psychostimulants, but not alcohol use, while adolescent cannabinoid exposure sensitizes an individual to opioid but not psychostimulant use. These drug-drug interactions are likely driven by the drug's direct neurobiological effect on neurodevelopmental processes, in addition to interactions with other factors, such as sex, genetic background, and other environmental influences that complicate these associations. Indeed, there is a complex interplay between genetic and environmental influences such that studies of discordant mono and dizygotic twins report that early initiation of alcohol use (and other commonly available drugs) is largely driven by environmental influences in early adolescence with a shift of genetics assuming increased importance across adolescent development (Dick et al, 2016). Recapitulating this complex interaction in animal models is challenging but will provide important neurobiological insights. 
Although not addressed in the initial gateway hypothesis landmark study, an increasing effort in the field has been focused on understanding the relevance of sex in addiction vulnerability. In general, human investigations indicate that males are more at risk for substance use disorders even though females are more sensitive to the reinforcing properties of drugs. For instance, it has been reported that adolescent alcohol exposure has a long-term effect into adulthood particularly for females with increased acquisition of cocaine self-administration behavior, but not in males (Mateos-Garcia et al, 2015). The data remains limited to make definitive conclusions regarding gateway-like effects in males and females to help discern sex-dependent relationships in relation to the progression of addiction.

Finally, an intriguing consideration that has come to attention in recent years is the aspect of transgenerational transmission. The possibility that early drug use might alter addiction risk in humans has inspired recent interest about the potential long-lasting impact not only within their lifetime but even across generations. One of the first studies in this regard related to paternal alcohol exposure in which it was demonstrated to influence the adult offspring in regard to their sensitivity (locomotor response) to amphetamine (Abel, 1993). In addition, our lab, which has focused on $\Delta^{9}$-tetrahydrocannabinol (the psychoactive component of cannabis), identified significant cross-generational effects insomuch that parental exposure during adolescence increases the subsequent adult offspring's heroin selfadministration behavior that coincided with disrupted striatal neuroplasticity and epigenetic reprogramming (Szutorisz and Hurd, 2016). Thus, cross-generational gateway-like effects may also be of relevant to understanding the vulnerability of drug addiction seen in adults.

Overall, the concept of the gateway hypothesis has inspired a large body of research, but there remain significant gaps of knowledge before we are able to fully accept or refute the hypothesis. Despite the growing number of published papers relevant to the gateway drug hypothesis, many complex factors still have not been thoroughly addressed to determine causality in animal models even excluding human-specific confounds that impact interpretation such as social, psychological, and legal considerations. As the legality regarding substances of abuse evolve, becoming more relaxed in many jurisdictions, understanding the effect of drug exposure during critical periods of neurodevelopment, particularly adolescence, is essential.

\section{FUNDING AND DISCLOSURE}

The authors declare no conflict of interest.

\section{ACKNOWLEDGMENTS}

YLH supported by NIH/NIDA grants DA030359 and DA033660 and MLM supported by NIH/NIDA Predoctoral Training grant F30-DA038954. Invited commentary on: 'Prior Exposure to Alcohol has no Effect on Cocaine SelfAdministration and Relapse in Rats: Evidence from a Rat Model that does not Support the Gateway Hypothesis' by Fredriksson et al (2016).

\section{REFERENCES}

Abel EL (1993). Paternal alcohol exposure and hyperactivity in rat offspring: effects of amphetamine. Neurotoxicol Teratol 15: 445-449.

Dick DM, Adkins AE, Kuo SI (2016). Genetic influences on adolescent behavior. Neurosci Biobehav Rev 70: 198-205.

Fredriksson I, Adhikary S, Steensland P, Vendruscolo LF, Bonci A, Shaham Y et al (2016). Prior exposure to alcohol has no effect on cocaine self-administration and relapse in rats: evidence from a rat model that does not support the gateway hypothesis. Neuropsychopharmacology (e-pub ahead of print).

Mateos-Garcia A, Manzanedo C, Rodriguez-Arias M, Aguilar MA, Reig-Sanchis E, Navarro-Frances CI et al (2015). Sex differences in the long-lasting consequences of adolescent ethanol exposure for the rewarding effects of cocaine in mice. Psychopharmacology 232: 2995-3007.

Spear LP (2016). Consequences of adolescent use of alcohol and other drugs: Studies using rodent models. Neurosci Biobehav Rev 70: 228-243.

Szutorisz H, Hurd YL (2016). Epigenetic effects of cannabis exposure. Biol Psychiatry 79: 586-594. 